\title{
Proton Beam Therapy for Prostate Cancer: An Overview
}

\author{
Suhag V1, Sunita BS ${ }^{2}$, Vats P $^{3}$ \\ 1MD DNB Radiation Oncology, Professor \& Head, Department of Radiation Oncology, \\ ${ }^{1}$ Command Hospital (SC) Pune, India \\ ${ }_{2}^{2}$ MD DNB Pathology, Professor Pathology, Armed Forces Medical College, Pune, India \\ ${ }^{3}$ Resident Radiation Oncology, Army Hospital (R\&R), Delhi Cantt, Delhi, India
}

\begin{abstract}
Patients diagnosed with localized prostate cancer have many curative treatment options including several forms of advanced conformal Radiotherapy. Proton radiation is one such radiation treatment modality and, due to its unique physical properties, offers the appealing potential of reduced side effects without sacrificing cancer control. Patients of proton beam therapy (PBT) for prostate cancer had been continuously growing in number due to its promising characteristics of high dose distribution in the tumor target and a sharp distal fall-off. While theoretically beneficial, its clinical values are still being demonstrated from the increasing number of patients treated with proton therapy, from several dozen proton therapy centers around the world. High equipment and facility costs are often the major obstacle for its wider adoption. The picture will be clearer in coming decade as more and more centers throughout the world avail access to this technique and more data emerges on PBT.
\end{abstract}

Keywords: Ca Prostate, proton beam therapy, toxicities, quality of life

\section{INTRODUCTION}

Major categories of radiotherapy (RT) for prostate cancer treatment are external beam RT (EBRT), and brachytherapy. EBRT can be performed using different techniques like three-dimensional conformal RT (3D-CRT), intensity modulated RT (IMRT), volumetric modulated arc therapy (VMAT), stereotactic body radiation therapy (SBRT), stereotactic radiosurgery (SRS) and intensity modulated proton therapy (IMPT), etc., using a variety of radiation delivery machines, such as a linear accelerator (Linac), Cyberknife robotic system, Gamma knife, Tomotherapy and Proton beam machine. Proton therapy is an emerging technology for providing radiation therapy to cancer patients. PBT exploits subatomic particles with mass rather than $\mathrm{x}$-rays to deliver radiation dose. It has gained increasing interest primarily due to its advantageous physical property. When passing through tissue, a proton deposits increasing dose slowly until reaching a sharp increase (Bragg peak) at its maximum depth of penetration, eliminating the exit dose seen with photons. The depth dose distribution of a proton beam makes it a preferable radiation modality as it reduces radiation to the healthy tissue outside the tumor, compared with conventional photon therapy. ${ }^{1-4}$

The initial data on PBT is mainly from Europe, Japan and in United States. But this facility is increasingly being made available in other parts of the world including South America, Middle East, India, and China. There is a call for high-level evidence to support its use in the setting of increased cost. Despite increasing use, proton therapy (PT) remains a relatively limited resource. ${ }^{5}$

\section{Physics of proton beam}

Protons are positively charged particles, with a large rest mass, that continuously lose energy through interactions with surrounding atomic electrons and nuclei in the materials that they traverse. For protons the rate of energy loss per unit path length, defined as stopping power increases as the proton slows down. This phenomenon causes a proton to lose a significant amount of its energy very rapidly just prior to it stopping. Therefore, proton dose deposition peaks at the end of the proton range, resulting in the creation of the Bragg peak. A unique characteristic of protons is the negligible dose that is deposited in the regions distal to the Bragg peak once the protons stop. The depth at which protons stop and create their respective Bragg peak is related to the initial energy of the proton. For clinical applications of proton radiation therapy, one can continuously adjust the proton energies as they enter the patient to allow the protons to deposit their dose within the tumor volume, while sparing tissues distal to that tumor. This is in stark contrast with photon beams, which attenuate in an exponential fashion and therefore always deposit dose distal to the tumor. ${ }^{6}$

\section{Biological aspects of PBT}

Protons have completely different dose distribution properties compared with photons, and have the potential to avoid most of the extra-target radiation, imparted by the acceleration system that gives protons a specific momentum that carries them into a body. After traveling a specified distance, the velocity is slowed by interactions associated with their mass and charge, and then stopped abruptly at a specific depth. This is the point at which the proton will interact with surrounding electrons, delivering its energy and causing ionization of molecules and radiation damage in the DNA of the target cell. Protons are characterized by low linear energy transfer radiation, and tissue damage is caused by single-strand DNA breaks, with sublethal radiation damage and potential radiation damage repair. The relative biological efficiency of the proton beam is generally considered to be 1.1. With pencil beam scanning, the interaction of dynamic delivery with a moving target must be evaluated and minimized. Overall, at the biological level, there remain several uncertainties in our understanding of the interaction between protons and human tissues. ${ }^{7}$

\section{Clinical evidence favouring PBT}

Ha B and colleagues performed a prospective phase II study to compare acute toxicity among five different hypo fractionated schedules using proton therapy. Eighty-two 
patients with T1-3bN0M0 prostate cancer were randomized to one of five arms: Arm 1, 60 cobalt gray equivalent (CGE)/20 fractions/5 weeks; Arm 2, 54 CGE/15 fractions/5 weeks; Arm 3, 47 CGE/10 fractions/5 weeks; Arm 4, 35 CGE/5 fractions/2.5 weeks; and Arm 5, 35 CGE/5 fractions/4 weeks. These arms were categorized into the moderate hypo fractionated (MHF) group (52 patients in Arms 1-3) and the extreme hypo fractionated (EHF) group (30 patients in Arms 4-5). At a median follow-up of 7.5 years, 7-year biochemical failure-free survival (BCFFS) was 76.2\% for the MHF group and $46.2 \%$ for the EHF group ( $p=0.005)$. The 7-year BCFFS of the MHF and EHF groups were 90.5 and $57.1 \%$ in the low-risk group ( $p=0.154) ; 83.5$ and $42.9 \%$ in the intermediate risk group ( $\mathrm{p}=0.018)$; and 41.7 and $40.0 \%$ in the high risk group $(\mathrm{p}=0.786)$, respectively. The authors concluded that the efficacy of EHF is potentially inferior to that of MHF and that further studies are warranted, therefore, to confirm these findings. ${ }^{8}$

Arimura T et al evaluated the efficacy and adverse events of PBT alone for prostate cancer patients $(n=218)$ with intermediate- and high-risk disease. Patients were treated with PBT following one of the following protocols: 74 Gray (GyE) with 37 fractions (fr) (74 GyE/37 fr), $78 \mathrm{GyE} / 39 \mathrm{fr}$, and $70 \mathrm{GyE} / 28 \mathrm{fr}$. The 5-year progression-free survival rate in the intermediate- and high-risk groups was $97 \%$ and $83 \%$, respectively $(p=0.002)$. The rate of grade 2 or higher late gastrointestinal toxicity was $3.9 \%$, and a significant increased incidence was noted in those who received the 78 GyE/39 fr protocol $(p<0.05)$. Grade 2 or higher acute and late genitourinary toxicities were observed in $23.5 \%$ and $3.4 \%$ of patients, respectively. The authors concluded that PBT monotherapy can be a beneficial treatment for localized prostate cancer. Furthermore, it can preserve the quality of life of these patients. ${ }^{9}$

Pan HY and colleagues compared the toxicities and cost of proton radiation with intensity-modulated radiotherapy (IMRT) for prostate cancer among men younger than 65 years of age with private insurance. A total of 693 proton therapy patients were matched to 3,465 IMRT patients. Proton therapy patients had a lower risk of composite urinary toxicity $(33 \% \mathrm{v} 42 \%$ at 2 years; $\mathrm{P}<.001)$ and erectile dysfunction $(21 \% \mathrm{v} 28 \%$ at 2 years; $\mathrm{P}<.001)$, but a higher risk of bowel toxicity $(20 \% \mathrm{v} 15 \%$ at 2 years; $\mathrm{P}=$ $.02)$. Mean radiation cost was $\$ 115,501$ for proton therapy patients and $\$ 59,012$ for IMRT patients $(P<.001)$. It was found that among younger men with prostate cancer, proton radiation was associated with significant reductions in urinary toxicity but increased bowel toxicity at nearly twice the cost of IMRT. ${ }^{10}$

Iwata $\mathrm{H}$ and coworkers conducted a multi-institutional retrospective survey of the long-term outcomes of PBT for prostate cancer in Japan. There were 215, 520, and 556 patients in the low-risk, intermediate-risk, and high-risk groups, respectively. The median follow-up period of surviving patients was 69 months (range: 7-107). Among all patients, $98.8 \%$ were treated using a conventional fractionation schedule and $1.2 \%$ with a hypofractionation schedule; $58.5 \%$ and $21.5 \%$ received neoadjuvant and adjuvant androgen deprivation therapy, respectively. The 5year biochemical relapse-free survival (bRFS) and overall survival rates in the low-risk, intermediate-risk, and highrisk groups were $97.0 \%, 91.1 \%$, and $83.1 \%$, and $98.4 \%$, $96.8 \%$, and $95.2 \%$, respectively. The incidence rates of grade
2 or more severe late gastrointestinal and genitourinary toxicities were $4.1 \%$ and $4.0 \%$, retrospectively. This study suggested that PBT is effective and well-tolerated for prostate cancer. ${ }^{11}$

Ho CK and colleagues investigated long-term outcomes for men $\leq 60$ years old treated with PBT alone in 254 patients. Patients received 76-82 Gy at 2 Gy/fraction or 70-72.5 Gy at $2.5 \mathrm{~Gy} /$ fraction. Median follow-up for the cohort was 7.1 years; 7-year biochemical-free survival was 97.8\%. Eight men experienced biochemical progression, including one who died of disease 9 years after treatment. Potency was $90 \%$ at baseline and declined to $72 \%$ at the first-year followup, but declined to only $67 \%$ at 5 years. Only $2 \%$ of patients developed urinary incontinence requiring pads. The bowel habits mean score declined from a baseline of 96 to 88 at 1 year, which improved over the following years to 93 at 5 years. This study concluded that young men with prostate cancer continue to have excellent results with respect to 7year biochemical control and 5-year erectile function, without clinically significant urinary incontinence 5 years after proton therapy. ${ }^{12}$

In another study (Nakajima $\mathrm{K}$ et al, 2018) to evaluate differences in acute toxicity among patients with localized Prostate cancer treated with either conventional fractionated proton therapy (CFPT) or HFPT, a total of 526 eligible patients were analyzed. The patients received 74 gray relative biological effectiveness equivalents [Gy (RBE)]/37 fractions for low-risk patients and $78 \mathrm{~Gy}$ (RBE)/39 fractions for intermediate- and high-risk patients in the CFPT group $(\mathrm{n}=254)$ and $60 \mathrm{~Gy}(\mathrm{RBE}) / 20$ fractions for low-risk and 63 Gy (RBE)/21 fractions for intermediateand high-risk patients in the HFPT group $(n=272)$. No grade $\geq 3$ acute toxicity was observed in either group. Among acute genitourinary toxicities, grade 2 rates were $15 \%$ $(\mathrm{n}=38)$ in CFPT and 5.9\% $(\mathrm{n}=16)$ in HFPT $(\mathrm{P} \leq 0.001)$. There were no significant differences in acute gastrointestinal toxicity between the two groups. This study demonstrated the safety of HFPT for localized Prostate cancer patients in terms of acute toxicity. ${ }^{13}$

\section{Conclusion}

Despite the favorable results, PBT for prostate cancer has been a subject of controversy over the past two decades. Because of its dosimetric advantages when compared to conventional radiation, PBT has the potential to improve the therapeutic ratio in the management of prostate cancer by decreasing toxicity and improving disease control. Nevertheless, its higher costs and the current lack of uniform evidence documenting improved clinical outcomes have led some to question its cost-effectiveness. ${ }^{14,15}$ Though most retrospective studies concluded that PBT can be a suitable treatment option for localized prostate cancer. Future research should include more patients and longer follow-up schedules to clarify the definitive role of PBT.

\section{References}

[1] Wang D. A critical appraisal of the clinical utility of proton therapy in oncology. Med Devices (Auckl) 2015; 8: 439-446. doi: 10.2147/MDER.S65594

[2] Deville C. Proton Beam Therapy. In: Jack H. Mydlo, Ciril J. Godec, eds. Prostate Cancer: Science and clinical practice, 2nd edn. Academic Press 2016: p423-31. Available from: (http://www.sciencedirect.com/ science/article/pii/B9780128000779000463) 
[3] Royce T], Efstathiou JA. Proton therapy for prostate cancer: A review of the rationale, evidence, and current state. Urol Oncol. 2018 . pii: S1078-1439(18)30460-5. doi: 10.1016/j.urolonc.2018.11.012.

[4] Podder TK, Fredman ET, Ellis RJ. Advances in Radiotherapy for Prostate Cancer Treatment. Adv Exp Med Biol. 2018;1096:31-47. doi: 10.1007/978-3-31999286-0_2.

[5] Woodhouse KD, Hwang WT, Vapiwala N, et al. Sociodemographic disparities in the utilization of proton therapy for prostate cancer at an urban academic center. Adv Radiat Oncol.2017;18;2(2):132-139. doi: 10.1016/j.adro.2017.01.004.

[6] Chhabra A, Langen K, Mehta MP. An overview of modern proton therapy. Chin Clin Oncol 2016;5(4):48. doi: 10.21037/cco.2016.05.06

[7] Tian X, Liu K, Hou Y, Cheng J, Zhang J. The evolution of proton beam therapy: Current and future status. Molecular and Clinical Oncology 2018; 8(1): 15-21. https://doi.org/10.3892/mco.2017.1499

[8] Ha B, Cho KH, Lee KH, et al. Long-term results of a phase II study of hypofractionated proton therapy for prostate cancer: moderate versus extreme hypofractionation. Radiat Oncol. 2019; 14(1):4. doi: 10.1186/s13014-0191210-7.

[9] Arimura T, Yoshiura T, Matsukawa K, Kondo N, Kitano I, Ogino T. Proton Beam Therapy Alone for Intermediate- or High-Risk Prostate Cancer: An Institutional Prospective Cohort Study. Cancers
(Basel). 2018;10(4). pii: E116. doi: 10.3390/cancers10040116.

[10] Pan HY, Jiang J, Hoffman KE, et al. Comparative Toxicities and Cost of Intensity-Modulated Radiotherapy, Proton Radiation, and Stereotactic Body Radiotherapy Among Younger Men With Prostate Cancer. J Clin Oncol. 2018 Jun 20;36(18):1823-1830. doi: 10.1200/JC0.2017.75.5371. Epub 2018 Mar 21.

[11] Iwata $H$, Ishikawa $H$, Takagi $M$, et al. Long-term outcomes of proton therapy for prostate cancer in Japan: a multi-institutional survey of the Japanese Radiation Oncology Study Group. Cancer Med. 2018 ;7(3):677-689. doi: $10.1002 /$ cam4.1350.

[12] Ho CK, Bryant CM, Mendenhall NP, et al. Long-term outcomes following proton therapy for prostate cancer in young men with a focus on sexual health. Acta Oncol. 2018;57(5):582-588. doi: 10.1080/0284186X.2018.1427886.

[13] Nakajima K, Iwata $H$, Ogino $H$, et al. Acute toxicity of image-guided hypofractionated proton therapy for localized prostate cancer. Int J Clin Oncol. 2018;23(2):353-360. doi: 10.1007/s10147-0171209-8.

[14] Hoshina RM, Matsuura T, Umegaki K, Shimizu S. A Literature Review of Proton Beam Therapy for Prostate Cancer in Japan. J Clin Med 2019, 8(1): 48 pii: E48. doi: 10.3390/jcm8010048.

[15] Bryant C, Henderson RH, Hoppe BS, et al. Controversies in proton therapy for prostate cancer. Chin Clin Oncol.2016 Aug;5(4):55. doi: 10.21037/cco.2016.08.02. 